\title{
Temperature Dependence of Collisional Energy Transfer in Highly Excited Aromatics Studied by Classical Trajectory Calculations
}

\author{
By U. Grigoleit, T. Lenzer and K. Luther \\ Institut für Physikalische Chemie der Universität Göttingen, Tammannstr. 6, \\ D-37077 Göttingen, Germany
}

Dedicated to Prof. Dr. Dr. h. c. mult. Jürgen Troe on the occasion of his 60th birthday

(Received May 8, 2000; accepted May 15, 2000)

\author{
Collisional Energy Transfer / Highly Excited Molecules / \\ Temperature Dependence / Trajectory Calculations / \\ First and Second Moments
}

\begin{abstract}
The temperature dependence of the gas-phase collisional relaxation of highly vibrationally excited aromatic molecules has been studied using large scale classical trajectory calculations. The investigations have focused on azulene collisions with different colliders (He, $\mathrm{Ar}$ and $\mathrm{N}_{2}$ ) as well as pyrazine self-collisions providing the moments of energy transfer $\langle\Delta E\rangle$ and $\left\langle\Delta E^{2}\right\rangle$ in the temperature range $50-1500 \mathrm{~K}$. The interaction well depth $\varepsilon_{\text {eff }} / k_{\mathrm{B}}$ is found to be the key factor controlling the observed $T$ dependence of collisional energy transfer. Systems with a relatively deep interaction well (pyrazine + pyrazine, azulene + $\mathrm{Ar}$, azulene $+\mathrm{N}_{2}$ ) show a pronounced negative dependence of $-\langle\Delta E\rangle$ when $T<\varepsilon_{\text {eff }} / k_{\mathrm{B}}$ (in the systems studied here roughly at $T<300-400 \mathrm{~K}$ ). The increased efficiency of collisional energy transfer at low $T$ is due to additional contributions from collisions at large impact parameters. In systems with a very shallow well (azulene $+\mathrm{He}$ ), however, a positive $T$ dependence is found in the low temperature regime $(<300 \mathrm{~K})$ due to the dominant contributions from impulsive, adiabatic collisions at short impact parameters. At higher temperatures $(T>300-400 \mathrm{~K})$ - when the temperature is well above $\varepsilon_{\text {eff }} /$ $k_{\mathrm{B}}-$ all systems behave qualitatively similar, showing only a very weak, slightly negative $T$ dependence, as long as one is still far away from thermal equilibrium.
\end{abstract}

\section{Introduction}

Collisional energy transfer (CET) between a highly excited molecule and the surrounding bath medium is well known as an elementary process of 
specific importance in various types of reaction dynamics, as, e.g., in gasphase unimolecular reactions and combination processes $[1,2]$. In practice, such reactions occur under a wide variety of temperature conditions reaching from $200 \mathrm{~K}$ or below in the upper atmosphere up to several thousand $\mathrm{K}$ which can easily be reached, e.g., in combustion systems. Understanding of CET over wide temperature ranges is thus not only of basic interest but is also strongly related to practical requirements of reaction kinetics.

The key quantity for characterizing CET at the high densities of states, which occur in most molecules at chemically significant energies, is the collisional transition probability $P\left(E^{\prime}, E\right)$ for a transition from energy $E$ to $E^{\prime}$. Knowledge of $P\left(E^{\prime}, E\right)$ allows to determine all further quantities of interest, as, e.g., the first and second moments of CET $\langle\Delta E\rangle$ and $\left\langle\Delta E^{2}\right\rangle$. Experimentally, detailed information on $P\left(E^{\prime}, E\right)$ over wide energy ranges has been obtained so far only by the technique of "Kinetically Controlled Selective Ionization (KCSI)" developed in our group [3-5], inter alia for the deactivation of azulene, toluene, pyrazine and pyridine by a large variety of colliders. These experiments have so far been carried out at room temperature, and modifications to apply the technique to a wider temperature range are in progress. In contrast, standard methods based, e.g., on ultraviolet absorption (UVA) [6-8] or infrared fluorescence (IRF) [9-11] detection, measure $\langle\Delta E\rangle$, the first moment of CET, and its energy dependence, but not complete $P\left(E^{\prime}, E\right)$ or higher moments. Such studies have been applied to a wide range of systems (see, e.g., Refs. [3, 12]). Here we want to focus on temperature dependent direct CET studies carried out so far for large molecules, relevant to the comparison with our trajectory calculations. In these kinds of experiments, the highly vibrationally excited molecules are initially prepared with an average vibrational energy $E$ which is the sum of the average thermal energy $E_{\mathrm{th}}$ at temperature $T$ and an initial excitation energy $E_{\text {exc }}$ typically provided by a laser pump pulse.

Pioneering direct CET studies of the temperature dependence in large molecules were carried out by Troe and co-workers for highly vibrationally excited toluene molecules ("toluene*") colliding with 18 different bath gases, ranging from helium to large alkanes, like $n$-octane $[6,13]$. Measurements at 300 and $850 \mathrm{~K}$ were performed, and the laser excitation energy (40322 and $32468 \mathrm{~cm}^{-1}$, respectively) was chosen in the way, that the average vibrational energy $E$ of the toluene* molecules was identical at both temperatures. $\langle\Delta E\rangle$ was found to be virtually temperature independent for monoatomic and diatomic bath gases within the accuracy of the experiments. For larger bath gases a slightly negative temperature dependence was found. Both results are in agreement with earlier findings from indirect CET experiments employing steady-state quenching measurements of cycloheptatriene photoisomerization [14].

Very recently, Luther and co-workers applied time-resolved UVA experiments to characterize the $T$ dependent CET of highly vibrationally 
excited biphenylene molecules with a variety of mono- and polyatomic colliders [15]. Biphenylene was initially prepared with $\overline{E_{\text {exc }}}=28490 \mathrm{~cm}^{-1}$, and temperatures between 333 and $523 \mathrm{~K}$ were used. When they compared the results for different temperatures at the same average vibrational energy they found a general negative temperature dependence of $-\langle\Delta E\rangle$ in this system (for rare gases, $\mathrm{N}_{2}, \mathrm{CO}_{2}, \mathrm{SF}_{6}$, ethane, $n$-butane and $n$-heptane as colliders), very weak in light rare gases but systematically increasing with the efficiency of the collider. These results are thus in reasonable agreement with the toluene data.

Temperature dependent relaxation data on azulene and pyrazine, the molecules on which we present trajectory calculations, exist to various extent. For azulene, the temperature dependence of CET has been studied in the Barker group by means of IRF detection for the colliders $\mathrm{N}_{2}(T=300-$ $525 \mathrm{~K})$ and azulene $(T=300-630 \mathrm{~K})$, at $E_{\text {exc }}=29665 \mathrm{~cm}^{-1}$ [16]. Troe and co-workers applied UVA detection to study azulene collisions with 22 different bath gases at $T=300-800 \mathrm{~K}\left(E_{\text {exc }}=16949 \mathrm{~cm}^{-1}\right)$ [7]. Most recently, further UVA measurements on the temperature dependence of azulene energy transfer have been made by the same group within extended studies over a wide pressure range from dilute gas-phase conditions to compressed supercritical fluids $\left(T=373-612 \mathrm{~K}, E_{\text {exc }}=18797 \mathrm{~cm}^{-1}\right.$ for the gas-phase experiments) [17]. All azulene studies in this temperature range found a slight decrease of $-\langle\Delta E\rangle$ with temperature, very similar to toluene and biphenylene. For pyrazine, the only temperature dependent experiments available so far are IRF measurements by Barker et al. on self-collisions $\left(T=254-414 \mathrm{~K}, E_{\text {exc }}=32468 \mathrm{~cm}^{-1}\right)$ [18]. Their results show a pronounced negative temperature dependence at the lower temperatures but seem to level off at $300 \mathrm{~K}$. Two different sets of experimental IRF data obtained with 1- and 2-color-detection show that these measurements have an absolute precision of not more than $25 \%$. In summary, the experimental picture emerging from the available temperature dependent direct CET studies of large aromatic molecules in general showed a slightly negative or constant dependence of $-\langle\Delta E\rangle$ on temperature for $T>300 \mathrm{~K}$, whereas at lower temperatures a more strongly negative $T$ dependence of $-\langle\Delta E\rangle$ was found in one system. As will be shown in this paper these findings are also fully supported by our trajectory calculations.

It should however be mentioned that also quite different temperature dependencies of CET have been reported, e.g., for deactivation of the triatomics $\mathrm{CS}_{2}[19,20]$ and $\mathrm{SO}_{2}$ [21], for which a full variation of $T$ dependencies in a range of $T^{ \pm n}(n \leq 1)$ were measured. But also for large polyatomic molecules, CET temperature dependencies quite different from those discussed above are claimed even in recent literature: They reach, e.g., from no $T$ dependence (over wide temperature ranges) [22] to strong positive dependence linear in $T[23,24]$. These types of data are usually derived from very indirect sources, e.g., analysis of thermally or chemically acti- 
vated systems in the regime of pressure dependent kinetics. However, in such backward evaluations even the use of a full master equation analysis does not prevent from severe problems arising from assumed, too simplified models of CET.

On the theoretical side, models for explaining the experimentally observed $T$ dependence in large molecule energy transfer are almost completely lacking. In this respect, the "Sequential Direct Encounter (SDE) model" of Nikitin and co-workers has to be mentioned as one attempt, which is based on the Landau-Teller model for CET of diatomics and takes into account the interplay between the positive $T$ dependence originating from the energy-gap law and the negative $T$ dependence related to the collision lifetime $[25,26]$. While some ideas in this model are very useful at least for a qualitative understanding of the observed experimental trends, a quantitative description is not possible, as will be discussed in more detail in a forthcoming publication [27].

As far as molecular dynamics (MD) simulations are concerned, trajectory calculations are able to provide a wealth of information on the fundamental mechanisms of CET [28-33]. Surprisingly, studies of the temperature dependence of large molecule CET in the literature are rare and have so far not been systematically confronted with the existing experimental data. The first studies of this type for large molecule CET were carried out by Gilbert and co-workers for collisions between azulene and xenon [32]. This study shed some light on the energy flow between the vibrational, rotational and translational degrees of freedom in this system, yet - due to the particular choice of initial conditions - did not provide results directly comparable to the experimental data mentioned above. Also, the intramolecular azulene force field in that study used extremely small values for the torsional barriers, resulting in much too low normal mode frequencies compared to experiment. This is especially important, as it is known that the CET efficiency is widely determined by the lowest frequency modes [28]. This issue will be further commented elsewhere [27]. In the only other trajectory study available for large aromatics, Lim investigated toluene-helium collisions at 60,150 and $300 \mathrm{~K}$ with excitation energies between 15000 and $41000 \mathrm{~cm}^{-1}$ [33]. The $T$ dependence of $-\langle\Delta E\rangle$ from these calculations shows no clear trend, although the majority of the data suggest an almost constant or slightly decreasing $-\langle\Delta E\rangle$ with increasing temperature, in agreement with the experiments mentioned above.

To provide more insight into the collision mechanisms behind the experimentally observed $T$ dependence we present here results of our most recent trajectory studies of CET between highly excited aromatic molecules and several bath gases. Azulene collisions with $\mathrm{He}, \mathrm{Ar}$ and $\mathrm{N}_{2}$, as well as pyrazine-pyrazine collisions have been investigated in detail. A full account of the various aspects of these calculations will be given in a forthcoming publication [27]. Here we want to focus on key observations common to 
the studies we have carried out so far. Specifically, we will be able to explain, e.g., qualitatively different shapes for the temperature dependence of $\langle\Delta E\rangle$ found in systems with colliders of various types of efficiency.

\section{Trajectory method}

The trajectory method employed here has already been extensively discussed in our earlier publications [28, 29, 34, 35]. Briefly, CET in a quasicontinuum of rovibronic states can be quantified by an energy-resolved rate coefficient $k\left(E^{\prime}, E\right)$ for a collision, where a vibrationally excited molecule, having an initial energy $E$, interacts with a bath gas molecule and ends up with energy $E^{\prime}$ :

$$
\begin{aligned}
k\left(E^{\prime}, E\right)= & \lim _{b_{\mathrm{m}} \rightarrow \infty}\left(\frac{8 k_{\mathrm{B}} T}{\pi \mu}\right)^{1 / 2} \pi b_{\mathrm{m}}^{2} \int_{0}^{\infty} \int_{0}^{b_{\mathrm{m}}} \frac{E_{\text {trans }}}{\left(k_{\mathrm{B}} T\right)^{2}} \exp \left(-\frac{E_{\text {trans }}}{k_{\mathrm{B}} T}\right) \frac{2 \pi b}{\pi b_{\mathrm{m}}^{2}} \\
& \times B\left(E^{\prime}, E ; E_{\text {trans }}, b\right) d b d E_{\text {trans }} .
\end{aligned}
$$

$E_{\text {trans }}$ represents the relative impact energy, and $b$ is the impact parameter. $b_{\mathrm{m}}$, the maximum impact parameter, can be interpreted as an effective hardsphere diameter. $B\left(E^{\prime}, E ; E_{\text {trans }} b\right)$ is the probability for energy transfer from energy $E$ to $E^{\prime}$ for given initial values of $E_{\text {trans }}$ and $b . k\left(E^{\prime}, E\right)$ may be expressed as the product of a collisional transition probability $P\left(E^{\prime}, E\right)$ and a collision number $Z(E)$. The moments $\left\langle\Delta E(E)^{n}\right\rangle$ and $k_{E, n}$ of $P\left(E^{\prime}, E\right)$ and $k\left(E^{\prime}, E\right)$, respectively,

$$
\begin{aligned}
\left\langle\Delta E(E)^{n}\right\rangle & =\int_{0}^{\infty}\left(E^{\prime}-E\right)^{n} P\left(E^{\prime}, E\right) d E^{\prime} \\
& =\frac{1}{Z(E)} \int_{0}^{\infty}\left(E^{\prime}-E\right)^{n} k\left(E^{\prime}, E\right) d E^{\prime}=\frac{1}{Z(E)} k_{E, n},
\end{aligned}
$$

are easily accessed by classical trajectory calculations. $\left\langle\Delta E(E)^{n}\right\rangle$ can be split up into vibrational $\left(\left\langle\Delta E_{\text {vib }}^{n}\right\rangle\right)$ and rotational $\left(\left\langle\Delta E_{\mathrm{rot}}^{n}\right\rangle\right)$ contributions. In this paper, $\langle\Delta E\rangle$ always denotes the vibrational quantity, which fully dominates the total $\langle\Delta E\rangle$. For details, see Ref. [28].

The potential function $V$ was made up from contributions of intramolecular and intermolecular terms $\left[V=V_{\text {intra }}(\mathrm{A})+V_{\text {intra }}(\mathrm{M})+V_{\text {inter }}(\mathrm{A}+\mathrm{M})\right.$, where $\mathrm{A}=$ azulene or pyrazine and $\mathrm{M}=\mathrm{He}, \mathrm{Ar}, \mathrm{N}_{2}$ or pyrazine]. A simple harmonic valence force field (VFF) was used for azulene and pyrazine constructed from individual contributions of harmonic stretches, bends, out-ofplane wags and torsions [28, 29, 34, 35]. The individual parameters (including optimized force constants and normal mode frequencies) can be found in the Appendix (Tables 1 and 2). Specifically, for azulene the optimization started with the VFF of Lim and Gilbert [31]. The force constants for the torsions were then optimized so that the two lowest experimental normal 
mode frequencies were reproduced best (other force constants remained unchanged). Note that the earlier azulene VFF used in the temperature dependent calculations of Clarke et al. employs unrealistically small torsional barrier heights [36]. This yields 111 and $131 \mathrm{~cm}^{-1}$ for the lowest frequencies, whereas with our current VFF we obtain 189 and $240 \mathrm{~cm}^{-1}$ in perfect agreement with the experimental values [37]. This is important, because the lowest frequency modes predominantly determine the amount of energy transferred [28]. The collider $\mathrm{N}_{2}$ was described as a Morse oscillator with $r_{\mathrm{e}}=1.0977 \AA, \omega_{\mathrm{e}}=2358.6 \mathrm{~cm}^{-1}, \omega_{\mathrm{e}} x_{\mathrm{e}}=14.3, D_{\mathrm{e}}=9.905 \mathrm{eV}$ and $B_{\mathrm{e}}=$ $1.998 \mathrm{~cm}^{-1}$ [38].

The intermolecular potential was represented as a sum of pairwise atomatom potentials. Individual atom-atom terms for the azulene interaction with $\mathrm{Ar}$ and $\mathrm{N}_{2}$ employed generalized LJ 12-6-4 terms [39]:

$$
V=4 \varepsilon_{\mathrm{X}-\mathrm{Y}}\left[\left(\frac{\sigma_{\mathrm{X}-\mathrm{Y}}}{r_{\mathrm{X}-\mathrm{Y}}}\right)^{12}-\left(\frac{\sigma_{\mathrm{X}-\mathrm{Y}}}{r_{\mathrm{X}-\mathrm{Y}}}\right)^{6}\right]-\frac{B_{\mathrm{X}-\mathrm{Y}}}{r_{\mathrm{X}-\mathrm{Y}}^{4}}
$$

with $\mathrm{X}=\mathrm{C}$ or $\mathrm{H}$ and $\mathrm{Y}=\mathrm{Ar}$ or N. $\varepsilon_{\mathrm{X}-\mathrm{Y}}$ and $\sigma_{\mathrm{X}-\mathrm{Y}}$ are the $\mathrm{LJ}$ well depth and radius, respectively, for the interaction between atom $\mathrm{X}$ and $\mathrm{Y}$. The azulene dipole was modeled by placing point charges on each $\mathrm{C}$ atom giving rise to charge - induced dipole interactions with the bath gas atoms Ar and $\mathrm{N}$ [31], characterized by the parameter $B_{\mathrm{X}-\mathrm{Y}}=\left(q_{\mathrm{X}}^{2} \alpha_{\mathrm{Y}}\right) /\left(8 \varepsilon_{0} \pi\right)$, where $q_{\mathrm{X}}$ is the charge on carbon atom $\mathrm{X}$ and $\alpha_{\mathrm{Y}}$ is the polarizability of the bath gas atom Y. For details about the LJ potentials see Tables 3 and 4 in the Appendix. For azulene + helium we used the same EXP-6-4 potential as in Ref. [34], having the form

$$
V=A_{\mathrm{X}-\mathrm{Y}} \exp \left(-\gamma_{\mathrm{X}-\mathrm{Y}} r_{\mathrm{X}-\mathrm{Y}}\right)-C_{\mathrm{X}-\mathrm{Y}} r_{\mathrm{X}-\mathrm{Y}}^{-6}-D_{\mathrm{X}-\mathrm{Y}} r_{\mathrm{X}-\mathrm{Y}}^{-4} .
$$

We have recently parametrized this potential on the basis of experimental scattering data [40]. It yields a much better agreement with experimental CET moments [34] compared to the LJ potentials used earlier [31]. For pyrazine + pyrazine we used a simple LJ potential [Eq. (3); $\mathrm{X}=\mathrm{C}$ or $\mathrm{H}$, $\mathrm{Y}=\mathrm{C}$ or $\mathrm{H}$; no $r^{-4}$ term]. Individual parameters for this potential can also be found in the Appendix (Table 3).

The initial conditions were selected in a fashion similar to our earlier calculations $[28,29]$, with the following specific values: The initial translational and rotational distributions were chosen from Boltzmann distributions at the desired translational and rotational temperature $\left(T=T_{\text {trans }}=\right.$ $T_{\text {rot }}$, azulene calculations: $50-900 \mathrm{~K}$, pyrazine calculations: $200-1500 \mathrm{~K}$ ). The collider $\mathrm{N}_{2}$ was assumed to be in $v=0$ (in addition, our calculations showed that even a substantial population of the $v=1$ level would have practically no effect on the CET quantities). The initial vibrational energy $E$ of pyrazine was fixed at $30000 \mathrm{~cm}^{-1}$, to be directly comparable with the 2-color IRF data from Ref. [18]. For azulene we used $E=5000$ and $15000 \mathrm{~cm}^{-1}$, and the results presented in this paper concentrate on the case 
$E=15000 \mathrm{~cm}^{-1}$. With increasing temperature the fraction of the thermal energy within this total vibrational energy is progressively increasing, and by that the "true" excess energy relative to thermal equilibrium is becoming smaller and smaller. This is especially important at higher temperatures, e.g., $T_{\text {vib }}=900 \mathrm{~K}$ corresponds already to an average thermal vibrational energy $\langle E\rangle_{\mathrm{th}}($ azulene $)=11644 \mathrm{~cm}^{-1}$, calculated via $\langle E(T)\rangle_{\mathrm{th}}=\sum_{i=1}^{s}\left(h v_{i} /\right.$ $\left.\left[\exp \left(h v_{i} / k_{B} T\right)-1\right]\right)$, which is valid for a canonical ensemble of harmonic oscillators at temperature $T$, with $s$ being the number of oscillators. For illustration, this dependence of $\langle E\rangle_{\mathrm{th}}$ (azulene) on $T$ can be found in Fig. 8 in the Appendix. We also carried out some calculations for azulene, were the excess energy above the average thermal vibrational energy $\langle E\rangle_{\mathrm{th}}$ was identical at all temperatures.

Note that we use a microcanonical vibrational distribution in our calculations whereas the distributions during the collision cascade, e.g., in KCSI experiments are typically nearly Gaussian [4], with a width depending on the collision partner. However, the error introduced by this approximation is negligible, especially with respect to the systematic trends under consideration, as we have tested in separate calculations where vibrational distributions of finite widths were used. The lowest temperature studied depends on the nature of the collider. At very low temperatures the formation of long-lived complexes is observed and becomes dominant, which technically limit our investigations down to values between $50 \mathrm{~K}$ (azulene*-helium) and $200 \mathrm{~K}$ (pyrazine*-pyrazine).

The maximum impact parameter $b_{\mathrm{m}}$ was carefully adjusted depending on the system and temperature used. For the individual trajectory sets it ranged from $9 \AA$ (azulene $+\mathrm{He}$ ) to $16 \AA$ (azulene $+\mathrm{Ar}$ ). Correspondingly, starting and finishing distances were chosen between $14-18 \AA$ and $16-$ $19 \AA$ A. Typically, $6000-12000$ trajectories were calculated for each set of initial conditions using a customized version of the computer program VENUS [41]. The zero point energy was included in all calculations.

\section{Results and discussion}

As usual in the field of CET we present our results in terms of the first and second moments of energy transfer, $\langle\Delta E\rangle$ and $\left\langle\Delta E^{2}\right\rangle$. This means that the $k_{E, n}$, the $n$th moments of the CET second order rate constants $k\left(E^{\prime}, E\right)$ [Eq. (1)] obtained from the trajectory calculations, need to be scaled by an appropriate collision number $Z(E)$ via Eq. (2). Usually, the energy independent Lennard-Jones (LJ) collision number $Z_{\mathrm{LJ}}$ is used for this purpose, which in units of $\mathrm{m}^{3} \cdot \mathrm{s}^{-1}$ is given by:

$$
Z_{\mathrm{LJ}}=\pi \sigma_{\mathrm{AB}}^{2} \sqrt{\frac{8 k_{B} T}{\pi \mu_{\mathrm{AB}}}} \Omega_{\mathrm{AB}}^{(2,2)^{*}} .
$$




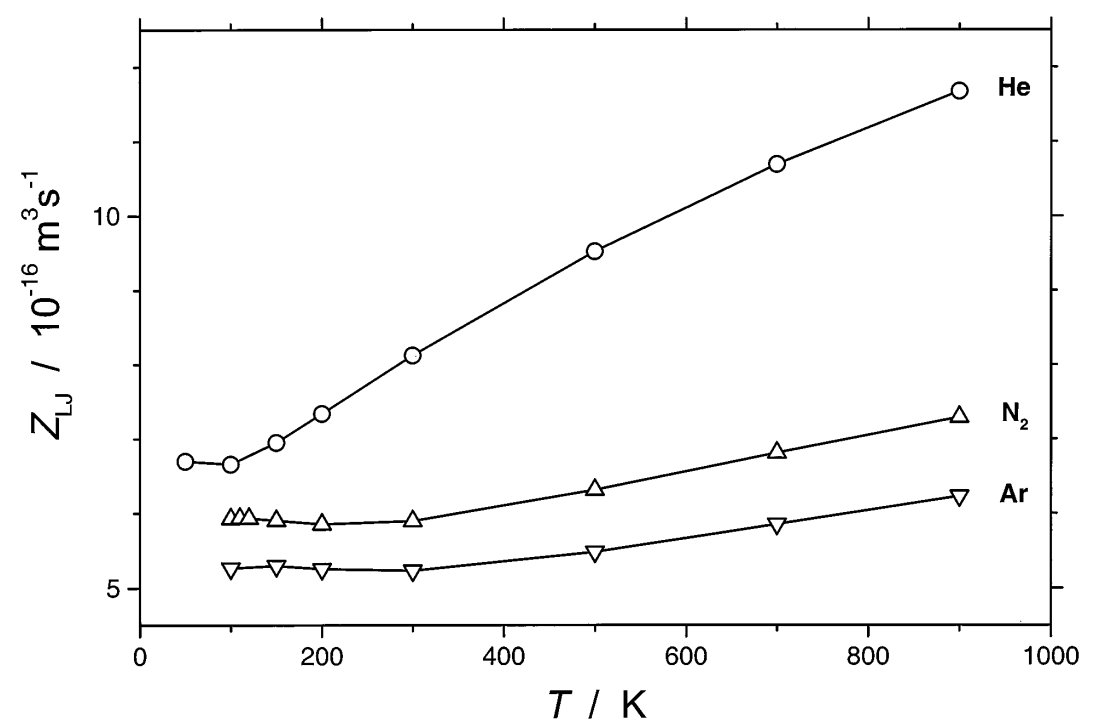

Fig. 1. Temperature dependent Lennard-Jones collision numbers $Z_{\mathrm{LJ}}$ for azulene $+\mathrm{M}$ $\left(\mathrm{M}=\mathrm{He}, \mathrm{Ar}\right.$ and $\left.\mathrm{N}_{2}\right)$.

$\sigma_{\mathrm{AB}}$ is the LJ radius (corresponding to $\sigma_{\mathrm{eff}}$ of Table 3 in the Appendix), $\mu_{\mathrm{AB}}$ represents the reduced mass of the collision pair, and $\Omega_{\mathrm{AB}}^{(2,2) *}$ is the collision integral taken from Ref. [42]. When using this parametrization, it is important to remember that part of the temperature dependence is already included in the collision number, with contributions from the velocity term $\left(\propto T^{1 / 2}\right)$ and the collision integral [42]. For illustration, Fig. 1 shows the temperature dependence of $Z_{\mathrm{LJ}}$ for the azulene-M collision pairs studied in this paper. $Z_{\mathrm{LJ}}$ increases most strongly with $T$ for azulene $+\mathrm{He}$, where nearly the full "hard sphere" dependence $\propto T^{1 / 2}$ is still retained, while much weaker $T$ dependencies occur due to the rather strong $\Omega_{\mathrm{AB}}^{(2,2)^{*}}(T)$ decrease for the larger colliders.

Fig. 2 shows our trajectory results for the temperature dependence of the first moment, $\langle\Delta E\rangle$, for highly vibrationally excited azulene molecules colliding with the bath gases $\mathrm{He}, \mathrm{Ar}$ and $\mathrm{N}_{2}$. The corresponding results for the second moment (plotted as the root mean squared average energy transferred per collision, $\left.\left\langle\Delta E^{2}\right\rangle^{1 / 2}\right)$ can be found in Fig. 3. In all these calculations, the azulene molecules had an initial vibrational energy of $15000 \mathrm{~cm}^{-1}$. Note that we have found totally analogous results at an excitation energy of $5000 \mathrm{~cm}^{-1}$ (not shown here). Experimental UVA results from Ref. [17] for He and Ar in the temperature range $T=373-612 \mathrm{~K}$ as well as KCSI results at $T=300 \mathrm{~K}$ [43] are also included for comparison 


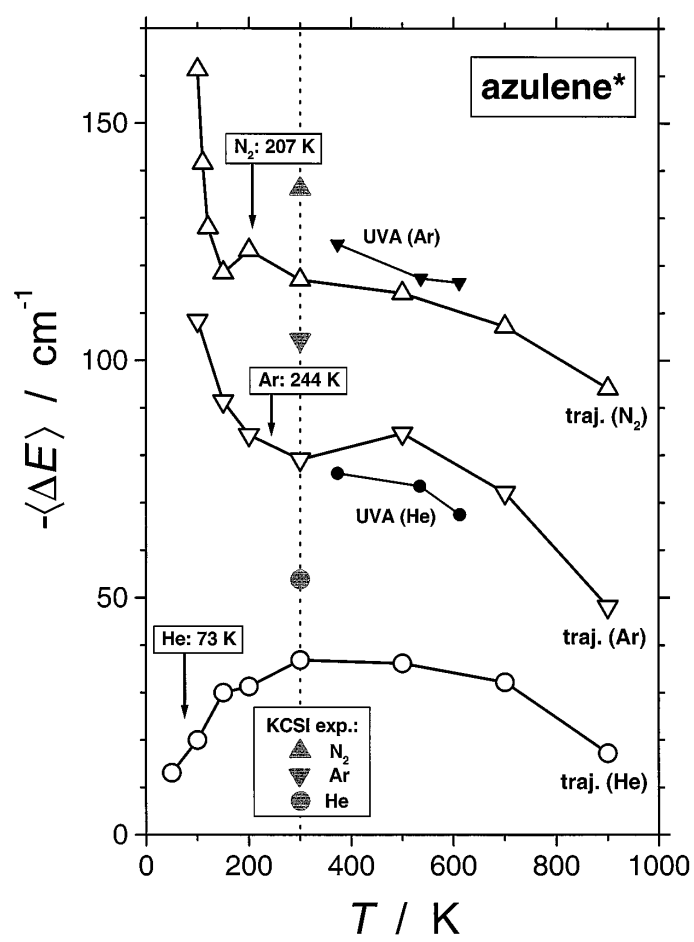

Fig. 2. Temperature dependence of $-\langle\Delta E\rangle$ for highly vibrationally excited azulene molecules colliding with different bath gases M. $T$ represents the translational and rotational temperature $\left(T=T_{\text {trans }}=T_{\text {rot }}\right)$. In all cases, the total vibrational energy of the azulene molecules is $E=15000 \mathrm{~cm}^{-1}$. He (circles), $\mathrm{Ar}$ (down triangles) and $\mathrm{N}_{2}$ (up triangles). Open symbols: results from the present trajectory calculations; filled black symbols: experimental UVA data for $\mathrm{He}$ and Ar from Ref. [17]; grey symbols: experimental KCSI results at $T=300 \mathrm{~K}$ from Ref. [43]. Arrows above each trajectory data set mark the effective well depth $\left(\varepsilon_{\text {eff }} / k_{\mathrm{B}}\right)$ for the respective azulene-M collision pair.

in the $\langle\Delta E\rangle$ plot. For $\left\langle\Delta E^{2}\right\rangle^{1 / 2}$, the only results available come from our own KCSI measurements.

The agreement found between experiment and trajectories is very satisfactory. Especially the azulene KCSI data at $300 \mathrm{~K}$, which might serve as the currently most accurate experimental benchmark results [3, 44], are in good agreement, although the trajectory results underestimate all experimental values slightly. A further improved quantitative agreement is possible by further "fine-tuning" of the intermolecular potentials as we have shown elsewhere [34]. For the study of the systematic effects in this paper, such further effort appeared not necessary.

The UVA results are consistently higher than the room temperature KCSI values, however also still close. A more detailed inspection of the 


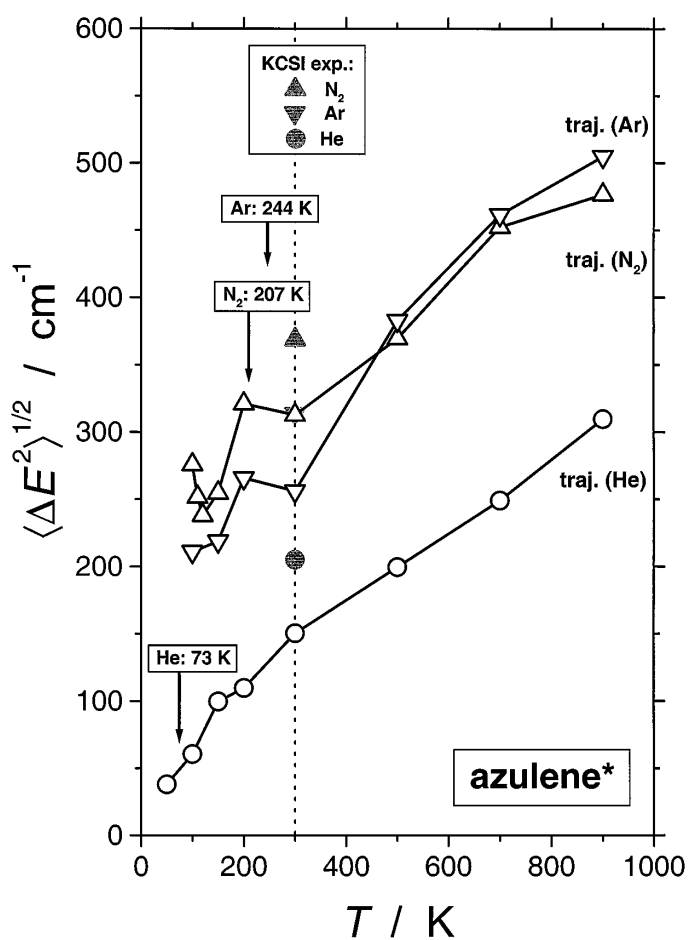

Fig. 3. Same as in Fig. 2, but for $\left\langle\Delta E^{2}\right\rangle^{1 / 2}$. The only experimental values available for comparison are the KCSI results at $T=300 \mathrm{~K}$ from Ref. [43]. Note that other standard CET methods, like, e.g., UVA or IRF can only provide $\langle\Delta E\rangle$ data, as those shown in Fig. 2.

trajectory results in Fig. 2 reveals that three ranges can be distinguished where the temperature dependence is different. At high temperatures $(>700 \mathrm{~K})$ a clearly negative $T$ dependence of $-\langle\Delta E\rangle$ is observed. This is the range where the initial vibrational energy of the azulene molecules $\left(15000 \mathrm{~cm}^{-1}\right)$ already approaches the average thermal vibrational energy [e.g., $\langle E\rangle_{\text {th }}($ azulene $)=11644 \mathrm{~cm}^{-1}$ at $900 \mathrm{~K}$ ], and the influence of "detailed balancing" becomes evident. Between about 300 and $700 \mathrm{~K}$ the $T$ dependence is weak, almost constant for helium and - on average - slightly negative for $\mathrm{Ar}$ and $\mathrm{N}_{2}$. Note that this is in agreement with the available UVA measurements in this temperature interval [17], also plotted in Fig. 2. The most interesting result is the behavior at very low temperatures. Strong negative dependencies of $-\langle\Delta E\rangle$ are observed especially for $\mathrm{N}_{2}$ (100$150 \mathrm{~K})$ and $\operatorname{Ar}(100-300 \mathrm{~K})$, whereas for helium an increase of $-\langle\Delta E\rangle$ with temperature is found in this range. 


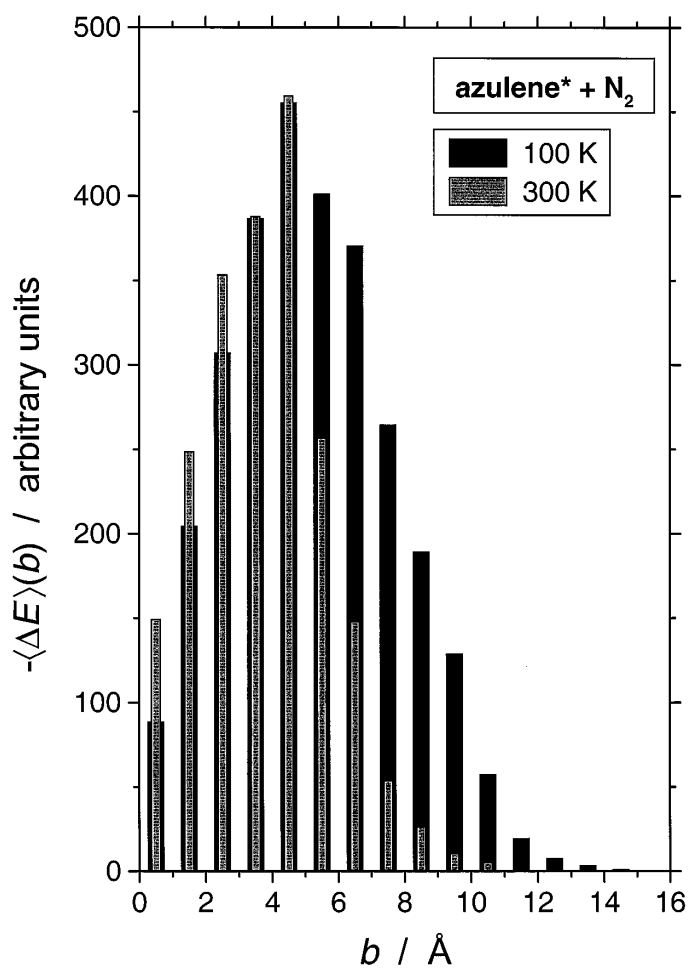

Fig. 4. Impact parameter resolved contributions to $\langle\Delta E\rangle$ for collisions between azulene* and $\mathrm{N}_{2}$ with initial temperatures of $T_{\text {trans }}=T_{\text {rot }}=100 \mathrm{~K}$ and $300 \mathrm{~K}$.

To rationalize these results it is very useful to have a closer look at the individual contributions to $\langle\Delta E\rangle$ from collisions at different impact parameters $b$. This is done in Figs. 4 and 5 for the systems azulene* $+\mathrm{N}_{2}$ and azulene $*+\mathrm{He}$, respectively, at two different temperatures (100 and $300 \mathrm{~K}$ ). In the case of $\mathrm{N}_{2}$ (and also for Ar) the increased CET efficiency at low $T$ is due to additional contributions from collisions at large impact parameters $(b>5 \AA)$, as is clearly seen by the larger $\langle\Delta E\rangle(b)$ amplitudes in this range. In contrast, at smaller $b$ the contributions at both low and high $T$ are very similar. This behavior can be explained as follows. At low temperature, the capture cross section for $\mathrm{N}_{2}$ is larger because the temperature is smaller than the effective interaction well depth $\varepsilon_{\text {eff }} / k_{\mathrm{B}}(207 \mathrm{~K}) . \mathrm{N}_{2}$ collisions starting at large $b$ can still result in close interactions and remove energy. At higher $T$ however, trajectories starting at large $b$ are deflected by the centrifugal angular momentum barrier. This picture is consistent with the fact that the increased CET efficiency for $\mathrm{N}_{2}$ and Ar shows up when $T<\varepsilon_{\text {eff }} / k_{\mathrm{B}}$ (see the downward pointing arrows in Figs. 2 and 3). 


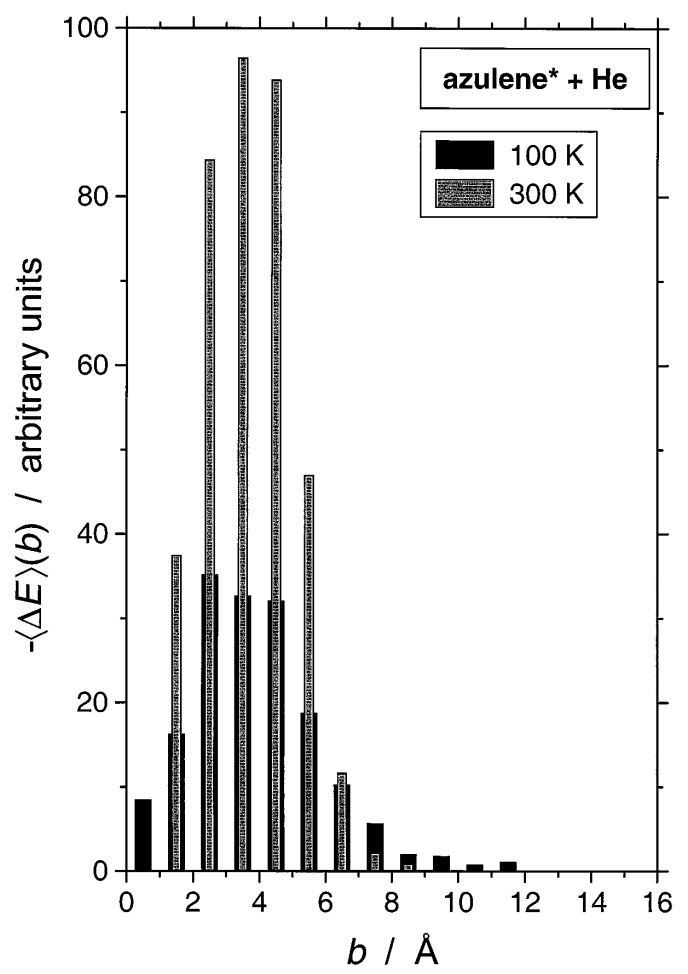

Fig. 5. Same as in Fig. 4, but for collisions between azulene* and He. Note the totally different appearance compared to azulene* $+\mathrm{N}_{2}$ in Fig. 4.

For helium the situation is completely different (Fig. 5). Here, the CET efficiency at higher $T$ is larger due to additional contributions from collisions at short impact parameters $(b=2-7 \AA)$. The explanation for this is, that even at $100 \mathrm{~K}$ the interaction well depth in the case of azulene* + $\mathrm{He}(73 \mathrm{~K})$ is still considerably smaller. Therefore the interaction is mainly repulsive and more "direct", impulsive collisions are dominant. Due to the "adiabaticity principle" the energy change in these collisions depends on the abruptness of the interaction and can be explained based on a "LandauTeller" type argument: Collisions at higher $T$ are more impulsive, and consequently the amount of energy transferred becomes larger.

In addition, we note - as a fine detail - that a similar effect is also found for $\mathrm{N}_{2}$ in Fig. 4 where collisions with small initial impact parameter $(b<4 \AA)$ are slightly more efficient at $300 \mathrm{~K}$ than at $100 \mathrm{~K}$. However, in that case the $T$ dependence is of course totally dominated by the - compared to $100 \mathrm{~K}-$ much more inefficient energy transfer at large $b$. In turn, in Fig. 5 the more efficient energy transfer of long range collisions at $100 \mathrm{~K}$ 


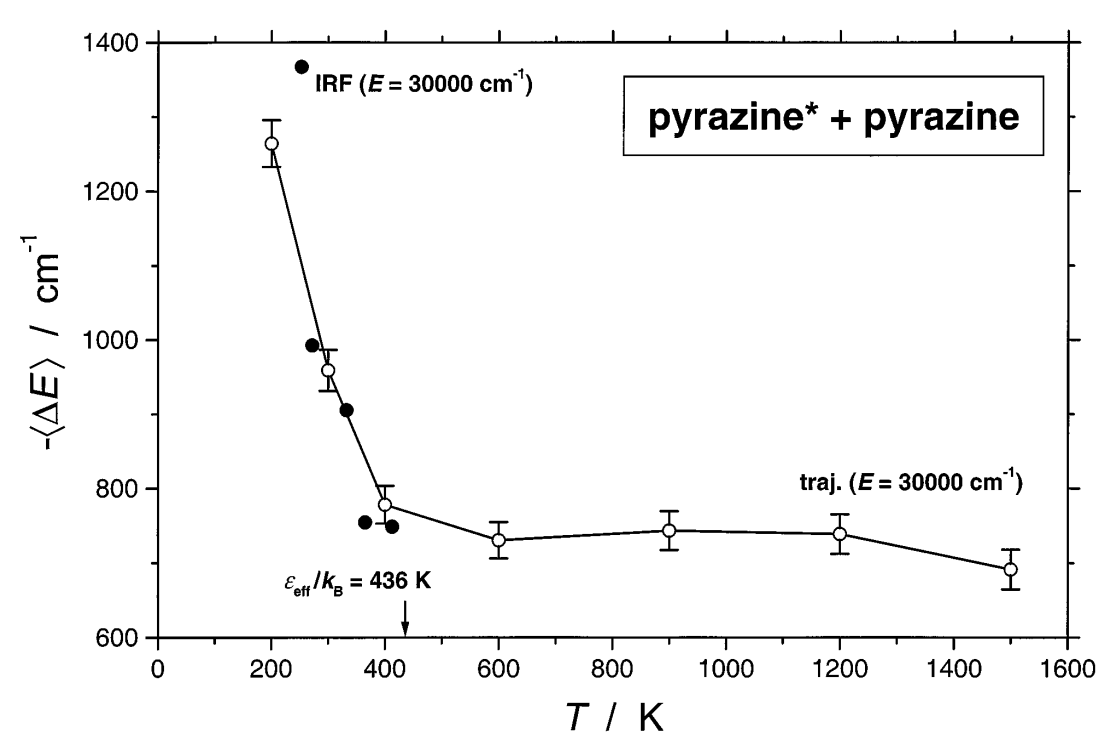

Fig. 6. Temperature dependence of $-\langle\Delta E\rangle$ for collisions between highly vibrationally excited pyrazine molecules and pyrazine. Open symbols with error bars: trajectory results from this work; black symbols: experimental results from Ref. [18]. Total vibrational energy $E=30000 \mathrm{~cm}^{-1}$. The effective Lennard-Jones well depth for pyrazine self-collisions $\left(\varepsilon_{\text {eff }} / k_{\mathrm{B}}=436 \mathrm{~K}\right)$ is marked by a downward pointing arrow.

is also found in the helium case $(b>7 \AA)$, however, there the much more efficient short range collisions at $300 \mathrm{~K}$ dominate the $T$ dependence.

As a final part of our discussion of the azulene results, we briefly want to inspect the temperature dependence of the second moment, plotted as $\left\langle\Delta E^{2}\right\rangle^{1 / 2}$ in Fig. 3. $\left\langle\Delta E^{2}\right\rangle^{1 / 2}$ shows a relatively uniform increase with $T$. Note, however, that a relatively sharp rise occurs for $\mathrm{N}_{2}$ at the lowest temperatures (below $120 \mathrm{~K}$ ), correlating with the trend observed for $-\langle\Delta E\rangle$. Fitting the $\mathrm{He}, \mathrm{Ar}$ and $\mathrm{N}_{2}$ results to a power law expression of the type $\left\langle\Delta E^{2}\right\rangle^{1 / 2} \propto T^{\gamma}$ yields values of $\gamma=0.70,0.44$ and 0.30 , respectively. This is in good agreement with earlier trajectory results from Lim for toluene* $+\mathrm{He}(\gamma=$ 0.44) [33]. A prediction of the SDE model of Nikitin et al. [25] yields $\gamma=$ 0.50 for a collision between a polyatomic molecule and an atom with a weak binding energy, like, e.g., helium, at not too low temperatures. In addition, for this case the model predicts $\langle\Delta E\rangle \approx$ const., which is also in good agreement with our helium calculations (compare, e.g., the range $300-700 \mathrm{~K}$ in Fig. 2).

We have found similar trends in our trajectory study of collisions between highly vibrationally excited pyrazine molecules colliding with the bath gases argon and pyrazine. For the latter case, Fig. 6 shows an example. The interaction between two pyrazine molecules is characterized by a well 


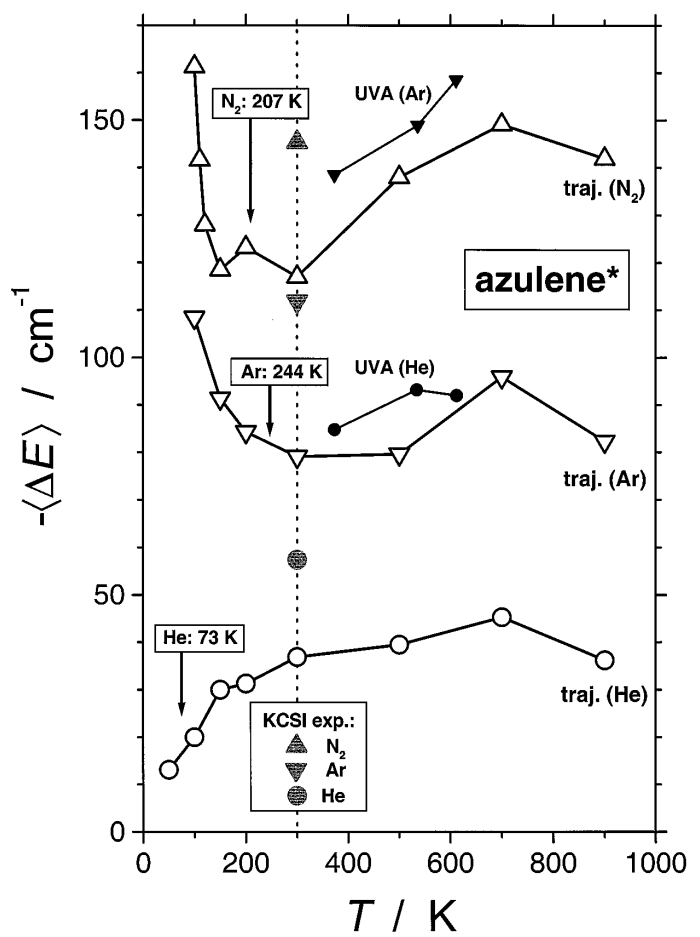

Fig. 7. Same as in Fig. 2, but in this case for an excess energy of $15000 \mathrm{~cm}^{-1}$ above the average thermal energy of the azulene molecules at the respective temperature. Note the different $T$ dependence compared to Fig. 2 at temperatures above $300 \mathrm{~K}$.

depth of $\varepsilon_{\text {eff }} / k_{\mathrm{B}}=436 \mathrm{~K}$, which is considerably deeper than for azulene $+\mathrm{Ar}$ and azulene $+\mathrm{N}_{2}$. One would therefore expect some extension of qualitatively similar trends, and this is indeed observed, as shown for $E=$ $30000 \mathrm{~cm}^{-1}$. The strongly negative $T$ dependence characteristic for the lowest temperatures is now found to reach up to about $T=400 \mathrm{~K}$, as one might conjecture on the basis of the roughly two times larger well depth compared with azulene- $\mathrm{N}_{2} /$ Ar. Moreover, we find full quantitative agreement with the experimental IRF results existing for the pyrazine* + pyrazine system [18]. At higher temperatures $(>400 \mathrm{~K})$ we observe the same change to a weak (slightly negative) $T$ dependence, which is again in full qualitative agreement with azulene $+\mathrm{Ar}$ and azulene $+\mathrm{N}_{2}$. The switch to a stronger negative $T$ dependence at even higher temperatures is, however, not yet observed, presumably because of the higher vibrational energy employed in these pyrazine calculations $\left(30000 \mathrm{~cm}^{-1}\right)$. As a consequence, one is still far away from thermal equilibrium even at $1500 \mathrm{~K}\left[\langle E\rangle_{\mathrm{th}}(\right.$ pyrazine, $1500 \mathrm{~K}) \approx$ 
$\left.13000 \mathrm{~cm}^{-1}\right)$ ], and one has an example for fixed total $E$ of a large molecule where influences of "detailed balancing" on $\langle\Delta E\rangle(T)$ are only minor even at the highest temperatures considered.

At this point one has to emphasize again, that the results in Figs. 2 and 3 at different temperatures are for the same total vibrational energy $E_{v i b}$. In Fig. 2 at $T>700 \mathrm{~K}$ one already observes a decrease of $-\langle\Delta E\rangle$, because the average thermal vibrational energy [e.g., $\langle E\rangle_{\mathrm{th}}($ azulene $)=11644 \mathrm{~cm}^{-1}$ at $900 \mathrm{~K}$, Fig. 8 in the Appendix] approaches the $15000 \mathrm{~cm}^{-1}$ initial total vibrational energy of the azulene molecules. At somewhat lower vibrational energy, e.g., $10000 \mathrm{~cm}^{-1}$, an even stronger decrease of $-\langle\Delta E\rangle$ would thus be seen crossing the zero value already below $900 \mathrm{~K}$. Such parts of $-\langle\Delta E\rangle(T)$ dependencies for constant total $E_{\text {vib }}$ do no longer contain information on collisional dyanmics but are demonstrations of statistical mechanics. In an alternative approach, which is also very close to many experimental conditions with constant excitation energy, one can compare results for an identical vibrational excess energy above the respective thermal equilibrium at each temperature (corresponding to different total vibrational energies). This is done in Fig. 7. At low $T(\leq 300 \mathrm{~K})$, as expected, there is no difference between the results in Figs. 2 and 7 as the average thermal energy is extremely low compared to the total excess energy, so that $E_{\text {exc }}+\langle E\rangle_{\text {th }}$ $\approx E_{\text {exc }}$. However, at higher $T(\geq 300 \mathrm{~K})$, for all bath gases the $T$ dependence changes completely, most clearly visible for $\mathrm{N}_{2}$ from $500-900 \mathrm{~K}$. In that case, the weakly negative temperature dependence observed in Fig. 2 changes into an increase of $-\langle\Delta E\rangle$ with $T$ in Fig. 7. In addition, as one is now at all temperatures far away from thermal equilibrium, the drop of $-\langle\Delta E\rangle$ from $700-900 \mathrm{~K}$ is much less pronounced than in Fig. 2.

In a very similar way, the temperature dependence of the experimental UVA results has completely changed from a weakly negative dependence (Fig. 2) to a positive dependence (Fig. 7). When discussing temperature dependences it is therefore important to state which energy basis is used, i.e., if total vibrational energies or excess vibrational energies above thermal equilibrium are considered in the experiments and calculations. For small molecules the distinction between the cases of $E_{\mathrm{vib}, \mathrm{tot}}=$ const. and $E_{\mathrm{vib}, \mathrm{exc}}=$ const. remains negligible in practice for the vibrational energies of interest up to $T$ far above $10^{4} \mathrm{~K}$, e.g., in triatomics. For larger polyatomics this distinction is however essential even for standard temperatures of high temperature reaction kinetics and an insufficient awareness of this point may have contributed to the confusion sometimes found in global statements on "the temperature dependence of energy transfer" at high temperatures.

\section{Conclusions}

Our classical trajectory calculations have drawn a clear and consistent picture regarding the temperature dependence of large molecule CET and can 
offer physically reasonable explanations of the observed trends. In addition, the findings agree completely with the existing body of experimental UVA, IRF and KCSI data, and show also satisfactory quantitative agreement.

At temperatures below the effective well depth of the systems under consideration $\left(T<\varepsilon_{\mathrm{eff}} / k_{\mathrm{B}}\right)$, a strong negative temperature dependence of the first moment of energy transfer, $-\langle\Delta E\rangle$, is observed, as clearly seen in our case studies of azulene* + Ar, azulene* $+\mathrm{N}_{2}$ and especially pyrazine* + pyrazine. The increased CET efficiency at low $T$ is due to additional contributions from collisions at large impact parameters. At low $T$ such glancing collisions can still result in close interactions and energy transfer, due to the fact that the capture cross section is larger because the temperature is smaller than the effective interaction well depth $\varepsilon_{\text {eff }} / k_{\mathrm{B}}$. In contrast, at higher $T$, trajectories starting at large $b$ are deflected by the centrifugal angular momentum barrier. For systems with a very small well depth (e.g., azulene* $+\mathrm{He}$ ) however, a positive $T$ dependence of $-\langle\Delta E\rangle$ is observed. The more efficient CET with increasing $T$ is due to additional contributions from collisions at short impact parameters. Because of the very shallow well, the interaction even at the lowest temperatures considered is mainly repulsive and "direct" impulsive collisions are dominant. The collisions happen in the adiabatic regime and are therefore more impulsive (and more efficient) with increasing $T$.

At higher $T(>300-400 \mathrm{~K})$ - when the temperature climbs above $\varepsilon_{\text {eff }} f$ $k_{\mathrm{B}}$ - all systems behave qualitatively similar, showing only a slightly negative $T$ dependence, as long as one is still sufficiently far away from thermal equilibrium. At even higher temperatures the effect of "detailed balancing" on $-\langle\Delta E\rangle$ causes uniformly stronger negative temperature dependencies, forcing $-\langle\Delta E\rangle$ to zero when $\langle E\rangle_{\mathrm{th}}$ reaches the constant internal energy considered. One should be aware that in larger molecules this occurs already at experimentally very common temperatures even for higher vibrational energies. As such decay of $-\langle\Delta E\rangle$ due to the approach to equilibrium at high temperatures is not related to collision dynamics but only to statistical mechanics, consideration of the alternative $\langle\Delta E\rangle(T)$ dependence for $E_{\mathrm{exc}}=$ const. can be more informative in such cases.

\section{Acknowledgements}

The authors are greatly indebted to Prof. Jürgen Troe for uncountable stimulating and fruitful discussions on all facets of collisional energy transfer over many years. Financial support of this work by the Deutsche Forschungsgemeinschaft [SFB 93 ("Photochemie mit Lasern", C16) and 357 ("Molekulare Mechanismen unimolekularer Prozesse", A7)] is gratefully acknowledged. 


\section{Appendix}

Table 1. Intramolecular potential parameters for the valence force field of pyrazine.

$r_{\mathrm{e}}$ : equilibrium bond lengths, $\Theta_{\mathrm{e}}$ : equilibrium bond angles, $f_{\mathrm{s}}$ : stretching force constants, $f_{\Theta}$ : bending force constants, $f_{\alpha}$ : wagging force constants, $V_{0}$ : torsional barriers; ip $=$ inplane, oop $=$ out-of-plane. A normal mode analysis based on the given geometry and force constants yields the following 24 vibrational frequencies of pyrazine in $\mathrm{cm}^{-1}: 3 \overline{72}$, $432,635,655,665,841,869,905,983,1052,1060,1070,1099,1128,1247,1293,1518$, $1612,1699,1746,3056,3058,3060,3061$.

\begin{tabular}{|c|c|c|}
\hline Potential term & $\begin{array}{l}\text { Equilibrium bond length/ } \\
\text { equilibrium bond angle }\end{array}$ & $\begin{array}{l}\text { Force constant/ } \\
\text { barrier height for torsion }\end{array}$ \\
\hline $\mathrm{N}-\mathrm{C}$ stretch & $r_{\mathrm{e}}=1.3376 \AA$ & $f_{\mathrm{s}}=6.400$ mdyn $\AA^{-1}$ \\
\hline C-C stretch & $r_{\mathrm{e}}=1.3968 \AA$ & $f_{\mathrm{s}}=6.640$ mdyn $\AA^{-1}$ \\
\hline C-H stretch & $r_{\mathrm{e}}=1.0831 \AA$ & $f_{\mathrm{s}}=5.076 \mathrm{mdyn}^{\circ} \AA^{-1}$ \\
\hline NCC bend (ip) & $\Theta_{\mathrm{e}}=122.18^{\circ}$ & $f_{\Theta}=1.300 \mathrm{mdyn} \AA \mathrm{rad}^{-2}$ \\
\hline CNC bend (ip) & $\Theta_{\mathrm{e}}=115.65^{\circ}$ & $f_{\Theta}=1.300$ mdyn $\AA \mathrm{rad}^{-2}$ \\
\hline $\mathrm{NCH}$ bend (ip) & $\Theta_{\mathrm{e}}=117.86^{\circ}$ & $f_{\Theta}=0.500$ mdyn $\AA \mathrm{rad}^{-2}$ \\
\hline $\mathrm{CCH}$ bend (ip) & $\Theta_{\mathrm{e}}=119.96^{\circ}$ & $f_{\Theta}=0.520$ mdyn $\AA_{0} \mathrm{rad}^{-2}$ \\
\hline $\mathrm{CCH} / \mathrm{NCH}$ wag (oop) & - & $f_{\alpha}=0.350$ mdyn $\AA \mathrm{rad}^{-2}$ \\
\hline $\mathrm{C}-\mathrm{C}$ torsion & - & $V_{0}=17.00 \mathrm{kcal} / \mathrm{mol}$ \\
\hline $\mathrm{C}-\mathrm{N}$ torsion & - & $V_{0}=17.50 \mathrm{kcal} / \mathrm{mol}$ \\
\hline
\end{tabular}

Table 2. Optimized barrier heights for the torsions around the bonds connecting atoms $a$ and $d$ in the VFF of azulene. Parameters for the torsions not given here can be deduced from symmetry considerations. Numbering of the atoms see Fig. 9. Other VFF parameters taken from Ref. [32] without any changes. A normal mode analysis based on the given geometry and force constants yields the following 48 vibrational frequencies of azulene in $\mathrm{cm}^{-1}: 189,240,327,353,373,404,430,463,600,603,667,753,759,807,876,923$, 974, 991, 1072, 1080, 1120, 1149, 1182, 1193, 1198, 1223, 1257, 1284, 1323, 1328, 1357, 1480, 1569, 1634, 1714, 1787, 1806, 1920, 1990, 2035, 3032, 3033, 3033, 3033, 3034, 3034, 3039, 3043.

\begin{tabular}{rrrrrrr}
\hline Atom $a$ & Atom $b$ & Atom $c$ & Atom $d$ & Atom $e$ & Atom $f$ & $V_{\text {o }}[\mathrm{kcal} / \mathrm{mol}]$ \\
\hline 1 & 9 & 11 & 2 & 3 & 12 & 16.192 \\
3 & 2 & 13 & 10 & 4 & 9 & 17.644 \\
10 & 3 & 9 & 4 & 5 & 14 & 16.644 \\
4 & 10 & 14 & 5 & 6 & 15 & 17.541 \\
5 & 4 & 15 & 6 & 7 & 16 & 14.843 \\
9 & 1 & 8 & 10 & 3 & 4 & 59.891 \\
\hline
\end{tabular}


Table 3. Lennard-Jones parameters for the systems azulene $+\mathrm{Ar}$, azulene $+\mathrm{N}_{2}$ and pyrazine + pyrazine. $\varepsilon_{\mathrm{X}-\mathrm{Y}}$ and $\sigma_{\mathrm{X}-\mathrm{Y}}$ are the atom-atom parameters entering Eq. (3). The additional parameters $B_{\mathrm{X}-\mathrm{Y}}$ needed for the azulene systems can be found in Table 4. $\varepsilon_{\mathrm{eff}}$ and $\sigma_{\text {eff }}$ are the effective collision parameters characterizing the total interaction between the polyatomic molecule and the bath gas. These were determined using the program SIGMON [39], by numerical integration using midordinate rule on a 6-point ( $\mathrm{Ar}$ ) or 36-point grid ( $\mathrm{N}_{2}$, pyrazine).

\begin{tabular}{|c|c|c|c|c|c|}
\hline $\begin{array}{l}\text { System/ } \\
\text { potential type }\end{array}$ & $\begin{array}{l}\text { Atom-atom } \\
\text { interaction }\end{array}$ & $\varepsilon_{\mathrm{X}-\mathrm{Y}} / k_{\mathrm{B}}[\mathrm{K}]$ & $\sigma_{\mathrm{X}-\mathrm{Y}}[\AA]$ & $\varepsilon_{\mathrm{eff}} / k_{\mathrm{B}}[\mathrm{K}]$ & $\sigma_{\text {eff }}[\AA]$ \\
\hline azulene $+\mathrm{Ar}$ & $\mathrm{C}-\mathrm{Ar}$ & 48.236 & 3.3777 & & \\
\hline LJ 12-6-4 & $\mathrm{H}-\mathrm{Ar}$ & 27.260 & 3.2327 & 243.64 & 5.0417 \\
\hline \multirow{2}{*}{$\begin{array}{l}\text { azulene }+\mathrm{N}_{2} \\
\text { LJ 12-6-4 }\end{array}$} & $\mathrm{C}-\mathrm{N}$ & 22.568 & 3.3896 & & \\
\hline & $\mathrm{H}-\mathrm{N}$ & 12.754 & 3.2274 & 207.09 & 5.1750 \\
\hline \multirow{3}{*}{$\begin{array}{l}\text { pyrazine }+ \text { pyrazine } \\
\text { LJ } 12-6\end{array}$} & $\mathrm{~N}-\mathrm{N}, \mathrm{N}-\mathrm{C}, \mathrm{C}-\mathrm{C}$ & 35.200 & 3.3558 & & \\
\hline & $\mathrm{N}-\mathrm{H}, \mathrm{C}-\mathrm{H}$ & 19.677 & 3.1952 & 436.19 & 5.3572 \\
\hline & $\mathrm{H}-\mathrm{H}$ & 11.000 & 3.0345 & & \\
\hline
\end{tabular}

Table 4. Parameters $B_{\mathrm{X}-\mathrm{Y}}$ for the charge - induced dipole interaction between the azulene $\mathrm{C}$ atoms and the bath gas atoms $\mathrm{Ar}$ and $\mathrm{N}$, needed in Eq. (3). Charges $q_{\mathrm{C}}$ on the carbon atoms are also given. Polarizabilities used: $\alpha(\mathrm{N})=1.10 \cdot 10^{-30} \mathrm{~m}^{3}, \alpha(\mathrm{Ar})=$ $1.6411 \cdot 10^{-30} \mathrm{~m}^{3}[45]$. Numbering scheme see Fig. 9 .

\begin{tabular}{llll}
\hline Azulene carbon atom & $\begin{array}{l}q_{\mathrm{C}} \\
{[e]}\end{array}$ & $\begin{array}{l}B_{\mathrm{C}-\mathrm{Ar}} \\
{\left[\mathrm{kcal} \cdot \mathrm{mol}^{-1} \cdot \AA^{4}\right]}\end{array}$ & $\begin{array}{l}B_{\mathrm{C}-\mathrm{N}} \\
{\left[\mathrm{kcal} \cdot \mathrm{mol}^{-1} \cdot \AA^{4}\right]}\end{array}$ \\
\hline $\mathrm{C}_{1}=\mathrm{C}_{3}$ & -0.0649 & 1.13990 & 0.76926 \\
$\mathrm{C}_{2}$ & -0.00176 & $8.38309 \cdot 10^{-4}$ & $5.65730 \cdot 10^{-4}$ \\
$\mathrm{C}_{4}=\mathrm{C}_{8}$ & +0.00544 & $8.00897 \cdot 10^{-3}$ & $5.40483 \cdot 10^{-3}$ \\
$\mathrm{C}_{5}=\mathrm{C}_{7}$ & +0.000525 & $7.45929 \cdot 10^{-5}$ & $5.03388 \cdot 10^{-5}$ \\
$\mathrm{C}_{6}$ & +0.00488 & $6.44494 \cdot 10^{-3}$ & $4.34934 \cdot 10^{-3}$ \\
$\mathrm{C}_{9}=\mathrm{C}_{10}$ & -0.00101 & $2.76072 \cdot 10^{-4}$ & $1.86306 \cdot 10^{-4}$ \\
\hline
\end{tabular}




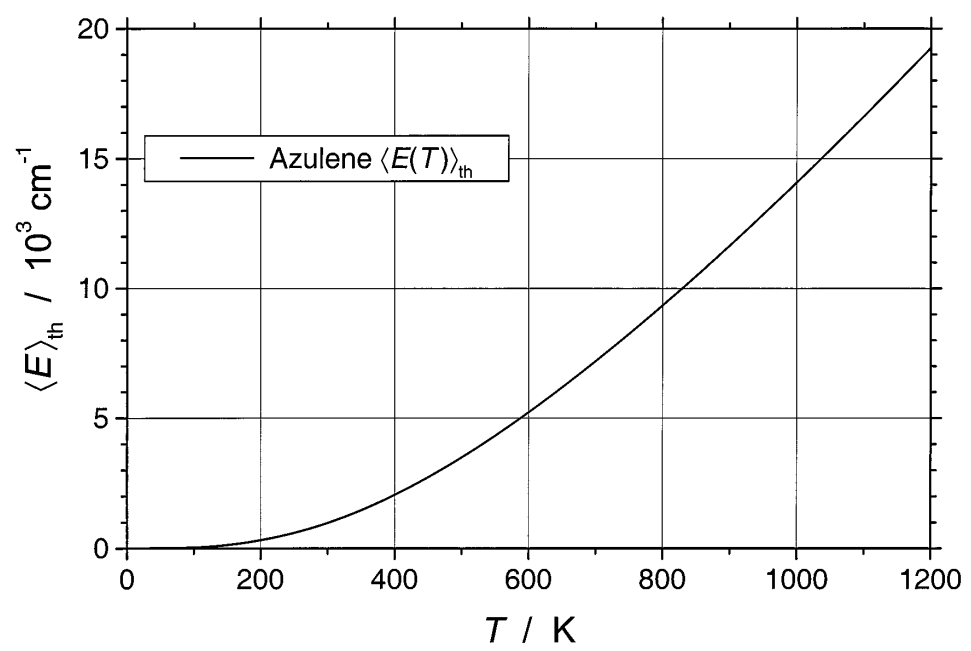

Fig. 8. Average thermal vibrational energy $\langle E\rangle_{\text {th }}$ of azulene as a function of temperature.

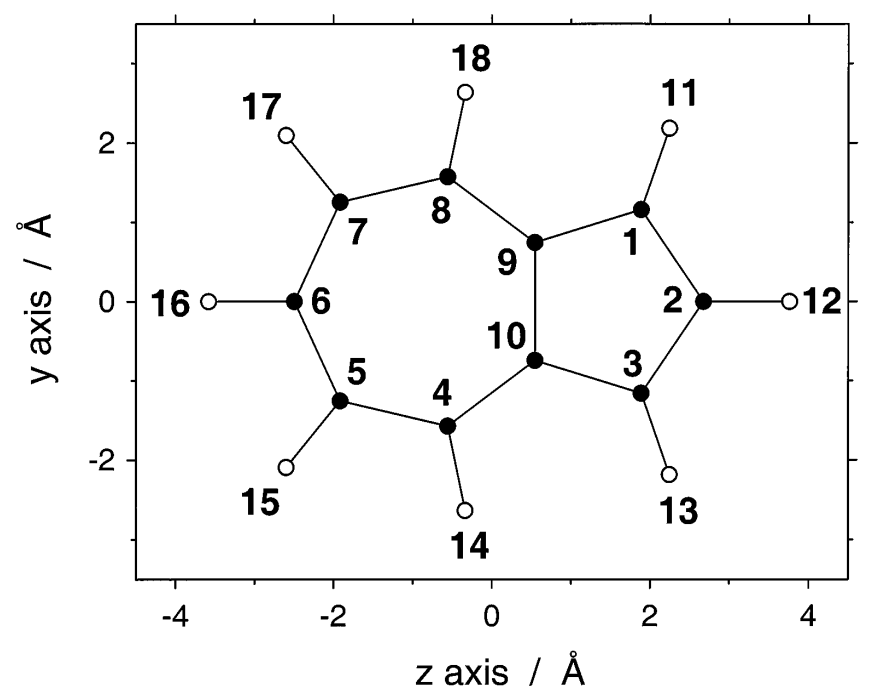

Fig. 9. Atom numbering scheme for azulene; see also Tables 2 and 4.

\section{References}

1. H. Hippler and J. Troe, in Bimolecular Collisions, eds. J. E. Baggott and M. N. R. Ashfold, The Royal Society of Chemistry, London (1989), p. 209.

2. M. Quack and J. Troe, in Gas Kinetics and Energy Transfer, Vol. 2, eds. P. G. Ashmore and R. J. Donovan, The Chemical Society, London (1977). 
3. U. Hold, T. Lenzer, K. Luther, K. Reihs and A. C. Symonds, J. Chem. Phys. 112 (2000) 4076.

4. T. Lenzer, K. Luther, K. Reihs and A. C. Symonds, J. Chem. Phys. 112 (2000) 4090.

5. K. Luther and K. Reihs, Ber. Bunsenges. Phys. Chem. 92 (1988) 442.

6. H. Hippler, J. Troe and H. J. Wendelken, J. Chem. Phys. 78 (1983) 6709.

7. H. Hippler, B. Otto and J. Troe, Ber. Bunsenges. Phys. Chem. 93 (1989) 428.

8. M. Damm, H. Hippler, H. A. Olschewski, J. Troe and J. Willner, Z. Phys. Chem. N.F. 166 (1990) 129.

9. J. Shi and J. R. Barker, J. Chem. Phys. 88 (1988) 6219.

10. B. M. Toselli, J. D. Brenner, M. L. Yerram, W. E. Chin, K. D. King and J. R. Barker, J. Chem. Phys. 95 (1991) 176.

11. L. A. Miller and J. R. Barker, J. Chem. Phys. 105 (1996) 1383.

12. I. Oref and D. C. Tardy, Chem. Rev. 90 (1990) 1407.

13. M. Heymann, H. Hippler and J. Troe, J. Chem. Phys. 80 (1984) 1853.

14. S. H. Luu and J. Troe, Ber. Bunsenges. Phys. Chem. 78 (1974) 766.

15. N. Fay and K. Luther, Z. Phys. Chem. Z. Phys. Chem. 214 (2000) 839.

16. J. R. Barker and R. E. Golden, J. Phys. Chem. 88 (1984) 1012.

17. C. Hanisch, University of Göttingen, Diploma Thesis (1997).

18. L. A. Miller, C. D. Cook and J. R. Barker, J. Chem. Phys. 105 (1996) 3012.

19. J. E. Dove, H. Hippler and J. Troe, J. Chem. Phys. 82 (1985) 1907.

20. M. Heymann, H. Hippler, H. J. Plach and J. Troe, J. Chem. Phys. 87 (1987) 3867.

21. M. Heymann, H. Hippler, D. Nahr, H. J. Plach and J. Troe, J. Phys. Chem. 92 (1988) 5507.

22. J. H. Kiefer and J. N. Shah, J. Phys. Chem. 91 (1987) 3024.

23. Y. Feng, J. T. Niiranen, A. Bencsura, V. D. Knyazev and D. Gutman, J. Phys. Chem. 97 (1993) 871

24. M. A. Hanning-Lee, W. J. Green, M. J. Pilling and S. H. Robertson, J. Phys. Chem. 97 (1993) 860.

25. E. I. Dashevskaya, E. E. Nikitin and I. Oref, J. Phys. Chem. 99 (1995) 10797.

26. E. I. Dashevskaya, E. E. Nikitin and I. Oref, J. Phys. Chem. 97 (1993) 9397.

27. T. Lenzer and K. Luther, Phys. Chem. Chem. Phys., to be published (2000).

28. T. Lenzer, K. Luther, J. Troe, R. G. Gilbert and K. F. Lim, J. Chem. Phys. 103 (1995) 626.

29. T. Lenzer and K. Luther, Ber. Bunsenges. Phys. Chem. 101 (1997) 581.

30. K. F. Lim and R. G. Gilbert, J. Phys. Chem. 94 (1990) 72.

31. K. F. Lim and R. G. Gilbert, J. Phys. Chem. 94 (1990) 77.

32. D. L. Clarke, I. Oref, R. G. Gilbert and K. F. Lim, J. Chem. Phys. 96 (1992) 5983.

33. K. F. Lim, J. Chem. Phys. 101 (1994) 8756.

34. T. Lenzer and K. Luther, J. Chem. Phys. 105 (1996) 10944.

35. T. Lenzer and K. Luther, J. Chem. Phys. 104 (1996) 3391.

36. W. D. Cornell, P. Cieplak, C. L. Bayly, I. R. Gould, K. M. Merz Jr., D. M. Ferguson, D. C. Spellmeyer, T. Fox, J. W. Caldwell and P. A. Kollman, J. Am. Chem. Soc. 117 (1995) 5179.

37. R. S. Chao and R. K. Khanna, Spectrochim. Acta 33A (1977) 53.

38. H.-J. Werner and P. J. Knowles, J. Chem. Phys. 94 (1991) 1264.

39. K. F. Lim, Program SIGMON: An Aid for the Semiempirical Fitting of the Intermolecular Potential, available from K. F. Lim, School of Biological and Chemical Sciences, Deakin University, Geelong, VIC 3217, Australia (1992).

40. R. Brandt, M. Henkel, B. Pfeil and W. Seidel, J. Chem. Phys. 95 (1991) 135.

41. W. L. Hase, R. J. Duchovic, X. Hu, A. Komornicki, K. F. Lim, D.-H. Lu, G. H. Peslherbe, K. N. Swamy, S. R. Vande Linde, A. Varandas, H. Wang and R. Wolf, QCPE 16 (1996) 671.

42. P. D. Neufeld, A. R. Janzen and R. A. Aziz, J. Chem. Phys. 57 (1972) 1100. 
43. U. Grigoleit, U. Hold, T. Lenzer, K. Luther and A. C. Symonds, J. Chem. Phys., to be published (2000)

44. U. Hold, T. Lenzer, K. Luther, K. Reihs and A. C. Symonds, Ber. Bunsenges. Phys. Chem. 101 (1997) 552

45. Handbook of Chemistry and Physics, 77 ed., CRC Press, Boca Raton (1996). 